\title{
HST IMAGING OF BETELGEUSE
}

\author{
RONALD L. GILLILAND \\ Space Telescope Science Institute \\ 3700 San Martin Drive, Baltimore, MD 21218, USA \\ AND \\ ANDREA K. DUPREE \\ Smithsonian Astrophysical Observatory \\ 60 Garden Street, Cambridge, MA 02138, USA
}

\section{Introduction}

We have obtained the first direct image of the surface of a star with $H S T$ in March 1995. ${ }^{1}$ Our near-ultraviolet images of the M2 Iab supergiantstar Betelgeuse (Alpha Orionis) taken with the COSTAR-corrected Faint Object Camera show about 10 resolution elements over the stellar disk. With direct resolution of Betelgeuse the following discoveries resulted: 1) The apparent size of the disk in the UV $(\sim \lambda 2550)$ is larger by a factor of $\sim 2.1$ than in the optical, demonstrating the extent of the supergiant atmosphere. 2) An unresolved hot spot (higher temperature than the disk by a few thousand degrees) dominates the spatial structure of the atmosphere. 3) The atmosphere at $2550 \AA$ extends to 125 milliarcsec (FWHM 108 mas) with a limb-darkening coefficient of about 1.0.

Observation of a stellar point source with the FOC shows a sharp point spread function (PSF) in which $\sim 9 \%$ of the total flux falls on the central 14.35 mas pixel. The PSF at $\lambda 2550$ has a FWHM of 38 mas. We note that the $H S T+$ FOC, operating in the ultraviolet has a resolution advantage over previous ground-based interferometry in the optical with $4-\mathrm{m}$ class telescopes. At $2550 \AA, \lambda / D(D=$ telescope diameter $)$ for the $2.4 \mathrm{~m}$ $H S T$ is smaller than that for the $4.2 \mathrm{~m}$ William Herschel Telescope (WHT)

\footnotetext{
${ }^{1}$ Based on observations with the NASA/ESA Hubble Space Telescope obtained at the Space Telescope Science Institute, which is operated by Association of Universities for Research in Astronomy, Incorporated, under NASA contract NAS5-26555.
} 
operated at 5460 , and $7100 \AA$ (Wilson et al. 1992 ) by $18 \%$ and $37 \%$ respectively. Further advantages follow from the intrinsically larger disk in the UV - about 125 mas at $2550 \AA$ as compared to $\sim 55$ mas at $5460 \AA$, and from the inherent simplicity afforded by direct images.

Betelgeuse has long been a favorite target for high-resolution imaging studies; especially since the discovery by Buscher et al. (1990) of a bright spot on the surface of Betelgeuse (in February 1989) through use of an interferometric technique (non-redundant masking) on the WHT. The same technique (Wilson et al. 1992) revealed two bright areas in February - April 1991. Surface structure and asymmetries in the extended atmosphere of Betelgeuse have also been suggested by polarimetric studies (Hayes 1984; Nordsieck et al. 1994).

While our FOC images fully exploit the spatial resolution capabilities of the $H S T$, the relatively broad-band filters at 2550 , and $2800 \AA$ with FWHM of 236 and $316 \AA$ respectively provide only marginal separation of $\mathrm{Mg}$ II line emission (only $10 \%$ of the F278M flux comes from Mg II) compared to continuum. We have also taken a large number of spectra with the G270M grating on GHRS covering $\sim 45 \AA$ at the Mg II lines. These spectra provide a spectrally pure separation of $\mathrm{Mg}$ II from the continuum and were acquired with some spatial information by stepping the 0.20 " "small" aperture by a series of 27.5 mas offsets over the stellar disk. Preliminary results from the GHRS spectra show that: 1) Mg II is more extended than the UV continuum, and 2) the outflow velocity is $\sim 10 \mathrm{~km} / \mathrm{s}$ at $2-3 R_{*}$.

In following sections we will review the observations and reductions (§2), discuss simple model fits and analyses applied to both the images and spectra to provide scales and velocities $(\S 3)$, and $\S 4$ will include preliminary interpretations and a summary.

\section{Observations and Data Reductions}

We have used both the FOC and the GHRS in relatively unique ways in order to derive information about Betelgeuse that requires data of both high-spectral and high-spatial resolution. The HST observations of Betelgeuse span 2 March 1995 (23:30 UT) to 3 March 1995 (15:00 UT).

\subsection{FAINT OBJECT CAMERA}

The FOC is the only HST instrument that provides adequate spatial sampling of its exquisite (corrected) near-UV PSF. To take advantage of this to image one of the brightest (and very red) stars on the sky required several steps: 1) A medium band filter (F253M or F278M) was crossed with a second UV filter, F220W. The red leak (light from the optical at $\lambda>$ $3100 \AA$ ) was thus suppressed to only $\sim 1-3 \%$ of the total even for this star 
in which the optical flux is 4 orders of magnitude above that for the near UV. 2) To allow a "high" count rate of $\sim 3$ counts/pixel/s with excellent linearity the small $128 \times 128$ mode format (1.84" square) was adopted. 3 ) To further reduce the expected count rate at least 4 magnitudes of neutral density filter were included. A separate exposure with an additional magnitude of neutral density filter allowed confirmation of perfect linearity for all exposures.

One exposure on Betelgeuse at $\sim 2500$ seconds was acquired for F253M + F220W and F278M + F220W each crossed with 4ND and 5ND filters separately. The 4ND and 5ND exposures (x2ml010[3-6]) allowed a direct solution for average detector dark count rates (found nominal values of $\sim 8 \times 10^{-4} \mathrm{cts} / \mathrm{px} / \mathrm{s}$ ), which was subsequently subtracted from the data. The real sky background reaching the detector through these stacked filters was effectively zero. A ratio of the $4 \mathrm{ND}$ and $5 \mathrm{ND}$ exposures returned a constant value of $\sim 2.5$ (to $\leq 1 \%$ ) independent of position in the PSF. With linearity assured the separate ND exposures were coadded (after verifying registration to $\leq 0.2$ pixel) yielding one final image at both F253M and F278M with peak intensities of 4833 and 7512 detected photons/pixel respectively in $\sim 3300$ s effective $4 \mathrm{ND}$ exposure time.

A PSF exposure (x2ml0303) of $2150 \mathrm{~s}$ with F278M + F220W was taken of the white dwarf, HZ 4 yielding a central intensity of 6334 counts.

The standard pipeline correction for geometric distortion was adopted. No correction for high spatial frequency flat-field structure was attempted, since the S/N of available flats $(\sim 20)$ fell far short of that attained in these images. Calibration flat-fields were carefully examined and no obvious structures exist near the stellar image position.

\subsection{GODDARD HIGH RESOLUTION SPECTROGRAPH}

GHRS spectra were taken with G270M covering 2776-2822 $\AA$. A series of 56 spectra with the 0.20 " SSA offset by different multiples of 0.0275 " from the stellar center were acquired on Betelgeuse. An integration time of 120 $s$ applied to each separate pointing. Overhead considerations forced use of the RAPID-READ (no substepping or comb-addition) mode in conjunction with spatial scans. A similar set of observations were acquired on $\eta \mathrm{UMa}$ to calibrate the expected response from a true point source.

A flat-field for this position on the photocathode was derived using an FP-SPLIT observation of $\eta$ UMa. All spectra were corrected for diode response differences, dark current, vignetting, linearity, and a derived flatfield. Data values at the positions of the five Side 2 dead diodes were set by linear interpolation from adjacent diodes. 
A
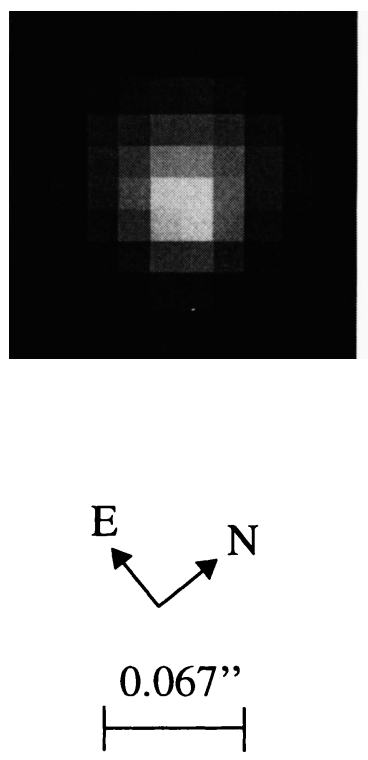

$10 \mathrm{AU}$
B

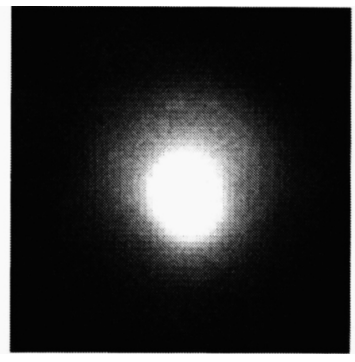

D

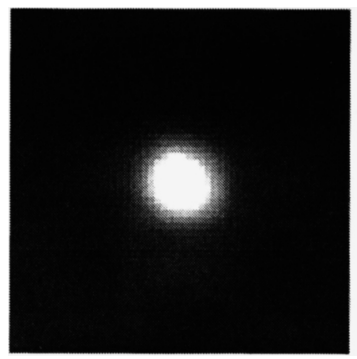

$\mathrm{C}$

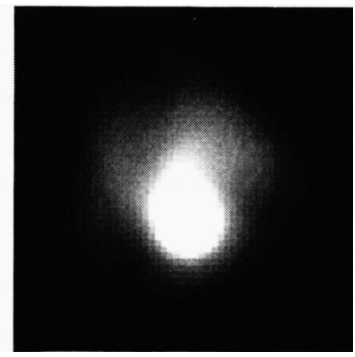

$\mathrm{E}$

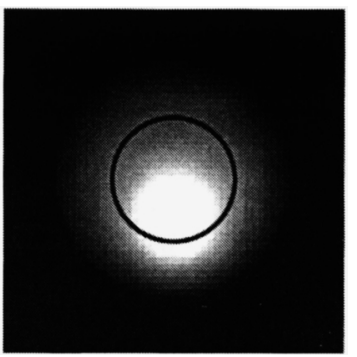

Figure 1. Three different representations of the F278M Betelgeuse image: (A) original data space, (B) over-sampled by a factor of five, (C) the latter deconvolved by the observed PSF. The HZ 4 PSF observation (D) after over-sampling. Panel E shows the hot spot plus limb-darkened disk model fit - over-sampled and deconvolved version, the superposed circle of 55 mas diameter shows the disk size as measured in the optical.

\section{Quantification of Scales and Velocities}

The FOC images provide the best information on spatial structure. Due to space limitations we will show results only for F278M; the F253M images are very similar. The GHRS spectra provide some spatial information, but for this brief discussion we will only discuss the high quality results on velocities.

\subsection{FOC IMAGES AND MODEL FITS}

The FOC images have been operated on by both over-sampling (bi-cubic spline interpolation by a factor of five) and deconvolution (used Jansson 1984 technique) for visualization purposes. Figure 1 shows the direct, oversampled, and over-sampled plus deconvolved images of Betelgeuse and for comparison an over-sampled F278M PSF image of HZ 4. The Betelgeuse image is to first order well represented by an extended disk plus a bright, off-center spot. Figure 2 compares radial intensity (in direct pixel space) 


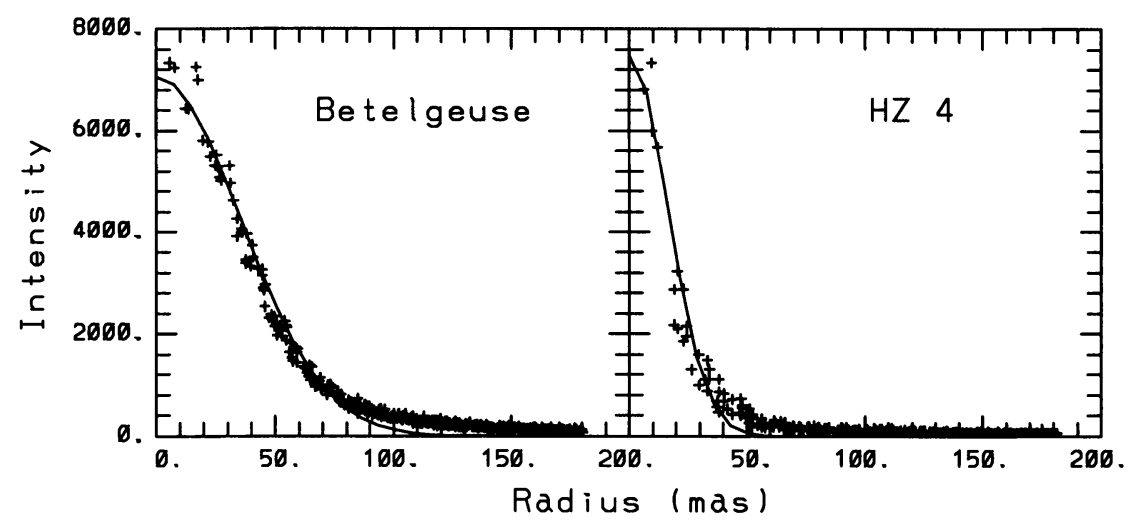

Figure 2. Radial intensity distributions of F278M images before over-sampling or deconvolution; curve in each case is a Gaussian fit with FWHM of 83 and 38 mas respectively.

plots for Betelgeuse and $\mathrm{HZ} 4$, clearly the former is well resolved.

We have performed non-linear least-squares fits of a two-component, limb-darkened disk plus gaussian hot spot, model to the Betelgeuse images. This model has 9 free parameters: amplitude, $\mathrm{x}$ and $\mathrm{y}$ position and width for both disk and hot spot and a standard limb-darkening coefficient for the disk. The results are very similar for analyses of direct pixel space, deconvolved single pixel, over-sampled, and deconvolved over-sampled images, thus lending confidence to the fidelity of the operations performed primarily for visualization purposes. Table 1 shows the model fit results.

TABLE 1. Betelgeuse Disk + Hot spot Model

\begin{tabular}{lccccc}
\hline Filter & $\begin{array}{c}\text { Hot spot } \\
\text { FWHM (mas) }\end{array}$ & $\begin{array}{c}\text { Disk } \\
\text { FWHM (mas) }\end{array}$ & $\begin{array}{c}\text { Separation } \\
\text { (mas) }\end{array}$ & $\begin{array}{c}\text { Amplitude } \\
\text { Hot spot }\end{array}$ & $\begin{array}{c}\text { Amplitude } \\
\text { Disk }\end{array}$ \\
\hline F253M & $31 \pm 2$ & $108 \pm 4$ & $16.5 \pm 3.4$ & $7750 \pm 900$ & $4400 \pm 200$ \\
F278M & $35 \pm 13$ & $96 \pm 4$ & $20.7 \pm 1.1$ & $8500 \pm 800$ & $6600 \pm 700$ \\
\hline
\end{tabular}

The errors listed are simply the internal scatter across fits to the four data representations. For comparison the FWHM of HZ 4 observed with F278M is 38 mas. Derived values of the limb-darkening coefficient varied from 0.8 to 1.1 (at $u=1.0$ a disk diameter of 125 mas corresponds to a FWHM of 108 mas). The separation gives the radial distance between the two model components.

A lower limit to the temperature enhancement of the spot is estimated from the $2550 \AA$ image by ratioing the total flux in the spot (as approxi- 


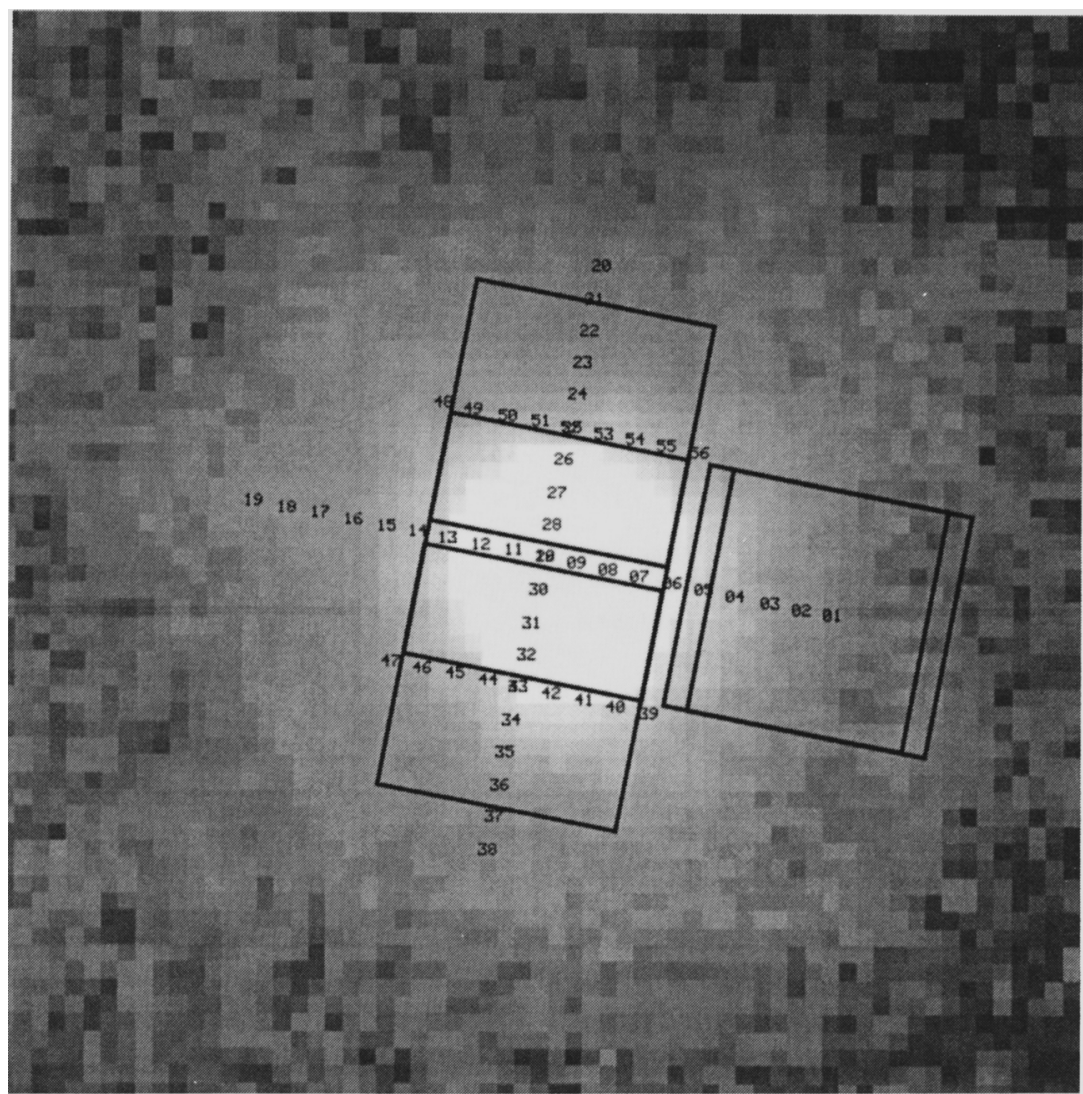

Figure 3. Pattern of GHRS 0.2" aperture (SSA) placements for Mg II region spectra of Betelgeuse. The boxes show the SSA size for a few illustrative pointings superposed to scale on the FOC F278M (logarithmic stretch) image.

mated by a Gaussian fit) to the total flux in the limb-darkened disk, and using the Planck law. The observed ratio corresponds to an excess of $\approx 2000 \mathrm{~K}$ at a temperature of $5000 \mathrm{~K}$ where the $2550 \AA$ continuum is formed.

\subsection{GHRS SPECTRA - RADIAL VELOCITY OF MG II}

Positions of the GHRS 0.2" aperture for the 56 independent spectra on Betelgeuse are shown in Figure 3. At the resolution provided by $H S T$ a 0.2 " aperture is obviously quite large. In principle it is possible to reconstruct the distribution of flux at each wavelength sampled by the GHRS on the spatial scale of the stepping. In practice such reconstructions are not competitive with the direct FOC images, although model fits to the GHRS data show good general consistency with the direct imaging results.

The GHRS spectra may be used to infer relative velocities at different 


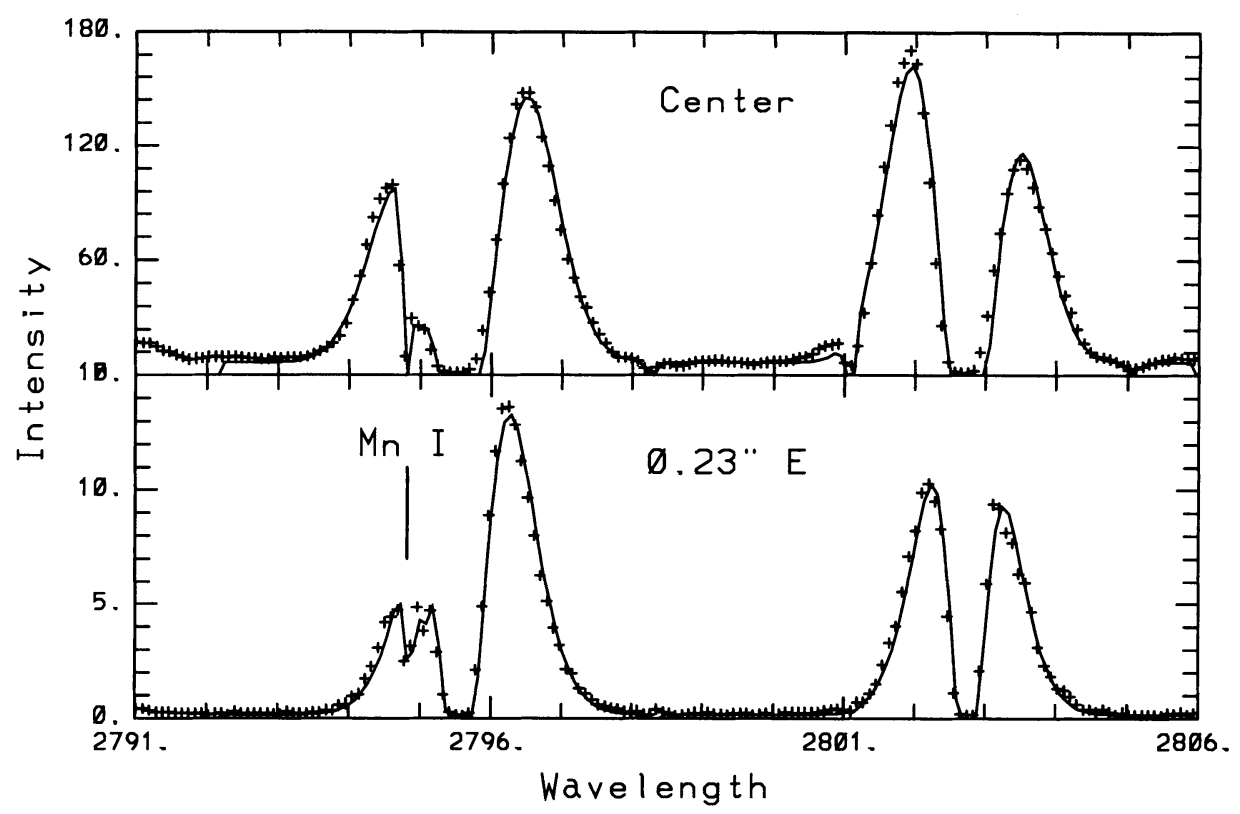

Figure 4. Spectra of Betelgeuse from positions 9 and 19 (see Fig. 3). The curves show a multi-gaussian fit including the $\mathrm{Mg}$ II emission lines, reversals, and some interstellar absorption lines. Note relative Mn I (2794.8 $\AA)$ and Mg II positions.

levels in the Betelgeuse atmosphere. Figure 4 shows a comparison of spectra acquired with the star centered in the aperture (scan no. 9) and with the aperture maximally offset from the stellar center (scan no. 19). A change in the relative positions of the $\mathrm{Mg}$ II emission component and sharp interstellar features is readily apparent near $2794.8 \AA$ (since the $\mathrm{Mg}$ II line widths change dramatically one must rely on model fit results even for qualitative comparisons).

Many factors influence the apparent velocity as observed with the GHRS: 1) aperture pulling - the weighted distribution of light in the dispersion direction relative to the aperture center, 2) geomagnetic image motion as the spectrograph changes position relative to the Earth's magnetic field, 3) thermal drifts in the spectrograph, 4) Doppler induced motions from $H S T$ 's changing velocity. All of these can be corrected for. A full reduction of velocities for the interstellar Mg II absorption lines in our point source comparison star, $\eta \mathrm{UMa}$ resulted in no dependence of velocity (good to an r.m.s. of $\sim 0.5 \mathrm{~km} / \mathrm{s}$ ) with aperture position relative to the star.

The offset spectra on Betelgeuse show a residual center-to-maximum offset velocity difference of $\sim 10 \mathrm{~km} / \mathrm{s}$ (Mg II lines are blue shifted at centered position) consistent with an expanding atmosphere. Also evident, but not yet fully analyzed is a large scale asymmetry in the velocities - perhaps as 
a result of rotation. Further analysis is required to extract all of the useful information on velocities.

\section{Interpretations and Summary}

Our imaging of Betelgeuse in the ultraviolet has opened a new chapter of stellar physics research. We highlight here a few of the potential scientific results.

Measurement of the size of the apparent diameter of the disk emission coupled with a determination of the limb-darkening provides parameters to model center-to-limb behavior at other wavelengths. A proper interpretation of interferometric measures of stellar diameters (Christou et al. 1988) depends on knowledge of the center-to-limb profile.

In showing one dominant bright feature the atmosphere of Betelgeuse is dramatically different from the UV image of the Sun (the only other star with a directly imaged disk) which typically has a mottled appearance arising from many active regions. We have evidence for a totally new physical phenomenon in luminous stellar atmospheres that promises to elucidate structure and energy balance in low gravity atmospheres. The discovery that the UV flux is dominated by a bright feature on one hemisphere of Betelgeuse can affect the ionization structure of the nearby material. Betelgeuse has a warm chromosphere and lacks dust within 900 mas radius (Danchi et al. 1994), so the radiation field will be an important factor in determining the structure of the circumstellar envelope (Huggins et al. 1994). Determination of the rotation vector of Betelgeuse from spatially resolved spectroscopy and/or motions of surface features may provide the link between observations of asymmetries in the envelope and circumstellar features.

We thank Drs. Peter Edmonds, Steve Hulbert and Han Uitenbroek for discussion and assistance with analyses. Excellent support by many members of the ST ScI, particularly the FOC group, in implementing these observations is gratefully acknowledged.

\section{References}

Buscher, D.F., Haniff, C.A., Baldwin, J.E., Warner, P.J., 1990, MNRAS, 245, 7P

Christou, J.C., Hebden, J.C., Hege, E.K. 1988, ApJ 327, 894

Danchi, W.C., Bester, M., Degiacomi, C.G., Greenhill, L.J., Townes, C.H. 1994, AJ 107, 1469

Hayes, D.P. 1984, ApJS 55, 179

Huggins, P.J., Bachiller, R., Cox, P., \& Forveille, T. 1994, ApJ 424, L127

Jansson, P.A. 1984, Deconvolution With Applications in Spectroscopy, (New York, Academic)

Nordsieck, K. et al., 1994, BAAS 26, 864

Wilson, R.W., Baldwin, J.E., Buscher, D.F., Warner, P.J. 1992, MNRAS 257, 369 